\title{
Incentives in Contests with Heterogeneous Solvers
}

\author{
Ersin Körpeoğlu \\ School of Management, University College London, London, UK, E14 5AB, e.korpeoglu@ucl.ac.uk \\ Soo-Haeng Cho \\ Tepper School of Business, Carnegie Mellon University, Pittsburgh, PA 15213, soohaeng@andrew.cmu.edu
}

\section{Forthcoming in Management Science}

\begin{abstract}
In a contest in which solvers with heterogeneous expertise exert effort to develop solutions, a recent paper (Terwiesch, C., Y. Xu. 2008. Innovation contests, open innovation and multiagent problem solving. Management Science. 54(9) 1529-1543) argues that as more solvers enter the contest, every solver will reduce effort due to a lower probability of winning the contest. This paper corrects mistakes in this theory, and shows that there exist high-expertise solvers who may raise their effort in response to increased competition. This is because more entrants raise the expected best performance among other solvers, creating positive incentives for solvers to exert higher effort to win the contest. Due to this positive effect, we find that a free-entry open contest is more likely to be optimal to a contest organizer than what Terwiesch and $\mathrm{Xu}$ (2008) and other prior literature asserted.
\end{abstract}

Key words: Contract; Crowdsourcing; Incentive; Tournament

Area of review: Operations Management

\section{Introduction}

Today many companies use contests (also called tournaments) as a mechanism for R\&D (research and development) outsourcing. A contest is a mechanism wherein a seeker poses a problem to a population of independent solvers, and awards the solver(s) that creates the best solution(s). A primary benefit of a contest is that a seeker can tap into a large number of experts outside of its firm boundary, and can select the most promising solution from many submitted solutions. However, merely collecting a large number of solutions does not necessarily guarantee the highest quality solution to a seeker. With many contest participants, solvers expect their individual chance of winning a contest to be low, and hence they may not have sufficient incentives to exert their 
best effort. Thus, a long-standing question in the contest literature has been "How do solvers' incentives to exert effort change with more participants in the contest?".

For contests in which solvers are heterogeneous in their expertise, Terwiesch and $\mathrm{Xu}$ (2008) (hereinafter T\&X) provide an answer to this question: Having more solvers in a contest leads to lower effort for every solver in equilibrium, because each solver's probability of winning decreases. This paper points out mistakes in the analysis of T\&X, and provides the correct analysis. Our analysis shows that there exist high-expertise solvers who may raise their effort in response to increased competition. This finding is in line with Moldovanu and Sela (2006) (hereinafter M\&S) who examine contests wherein solvers are heterogeneous in their costs of exerting effort. ${ }^{1}$ We prove this result in a unifying model that encompasses the models of M\&S and T\&X. We further articulate the drivers for this result: In addition to the reduced probability of winning, more entrants raise the expected performance of a runner-up, and therefore solvers have incentives to exert higher effort to win the contest. This second driver provides an opposing force to the negative externality created by increased competition. As a result, depending on which driver dominates the other, solvers react to increased competition differently. From a seeker's perspective, higher effort from high-ability solvers caused by increased competition is helpful to obtain a better solution from a contest. Therefore, we find that a free-entry open contest that allows the entry of all solvers is more likely to be optimal than what T\&X asserted. Specifically, whereas T\&X show that when solvers are heterogeneous in expertise, an open contest can be optimal under a certain condition, our result indicates that an open contest is optimal under a weaker condi-

\footnotetext{
${ }^{1}$ M\&S's model has some similarities to all-pay auctions in the sense that bidders (analogous to solvers) with heterogeneous valuations from winning an auction (similar to cost of effort) make bids (analogous to effort) to win the auction. In all-pay auctions, for example, Hopkins and Kornienko (2007) show that when a distribution for valuation increases in the likelihood ratio order, high-valuation bidders increase their bids, whereas low-valuation bidders reduce their bids. However, in all-pay auctions, an auctioneer maximizes the total bids of all bidders, whereas a contest organizer is interested in obtaining the best solution(s).
} 
tion than that of T\&X. ${ }^{2}$ This finding helps to justify the increased popularity of open innovation initiatives today.

\section{Model}

Consider a contest in which a seeker ("he") elicits solutions to a specified problem from a set of $n$ solvers ("she"). Each solver's performance $v$ is determined based on three components: solver's effort, expertise, and productivity. First, each solver $i$ can enhance her performance by investing in effort $e_{i}$ at the unit cost $c_{i}$. Second, each solver $i$ is endowed with initial expertise $\beta_{i}$, which measures prior knowledge and experience that will help the solver develop a solution to the problem posed by the seeker. Third, each solver $i$ is endowed with productivity $a_{i}$; given $e_{i}$, a solver with a higher $a_{i}$ achieves better performance than a solver with a lower $a_{i}$. Given her effort $e_{i}$, expertise level $\beta_{i}$, and productivity level $a_{i}$, solver $i$ 's performance is $v_{i}\left(\beta_{i}, a_{i}, e_{i}\right)=\beta_{i}+r\left(a_{i} e_{i}\right)$, where $r$ is an increasing and concave effort function.

Based on the performance vector $\left(v_{1}, \ldots, v_{n}\right)$, the seeker's payoff $V$ is determined as a weighted combination of the performance of the best solution and the average performance of all solutions: ${ }^{3}$

$$
V=\rho \max _{i=1, \ldots, n} v_{i}+(1-\rho) \frac{\sum_{i=1}^{n} v_{i}}{n}, \text { where } \rho \in[0,1] .
$$

The seeker's profit $\Pi$ is his payoff $V$ less the total amount of awards paid to solvers $A$; i.e., $\Pi=$ $V-A$.

${ }^{2}$ Contrary to our result, prior literature suggested that restricting the number of participants is optimal to a seeker. Taylor (1995) considers a contest among a pool of identical solvers, and Fullerton and McAfee (1999) analyze a contest in which a seeker auctions entry into a contest. These two papers show that more solvers in a contest will lead to lower effort for every solver, and they conclude that the number of participants should be restricted. However, in line with our result, Ales et al. (2016a) show that when the performance of solutions created by a pool of identical solvers is highly uncertain, an open contest is optimal.

${ }^{3}$ We utilize the payoff structure in (1) that is proposed by Terwiesch and Xu (2008) in order to compare our results with theirs. Moldovanu and Sela (2006) consider a special case of (1) where $\rho=1$ and another case where the seeker's payoff consists of the total performance of all solutions. Our model subsumes the former case and our results continue to hold in the latter case. 
The seeker makes two decisions. First, the seeker determines whether to have an open contest which allows any solver to participate in the contest. If the seeker chooses to restrict entry to the contest, $n$ becomes the number of solvers that are allowed to enter. Second, the seeker decides on a vector of awards $\left(A_{1}, A_{2}, \ldots, A_{n}\right)$, where $A_{j}$ is the award given to the solver with the $j$-th highest performance and $A=\sum_{j=1}^{n} A_{j}$. A solver with the best performance is referred to as the "winner," and a contest that awards only the winner (i.e., $\left.A_{1}=A\right)$ is called a "winner-take-all" contest.

An open contest proceeds in the following sequence. First, the seeker announces the vector of awards $\left(A_{1}, A_{2}, \ldots, A_{n}\right)$. Then, each solver $i \in\{1,2, \ldots, n\}$ privately learns her ability level (cost $c_{i}$, expertise $\beta_{i}$, and productivity $a_{i}$ ), and she determines whether to participate in the contest and her effort level $e_{i}$. If solver $i$ chooses not to participate in the contest, then she receives reservation utility 0 . If she chooses to participate, she incurs a fixed cost $k(\geq 0)$, and creates a solution by exerting effort $e_{i}$ at the unit $\operatorname{cost} c_{i}(>0)$. The seeker collects the solutions of all participating solvers, and he gives awards to solvers.

In a symmetric Bayesian Nash equilibrium, a solver with ability level $\left(c_{i}, \beta_{i}, a_{i}\right)$ chooses her equilibrium effort according to the function $e^{*}\left(c_{i}, \beta_{i}, a_{i}\right)$, and creates a solution with performance $v^{*}\left(c_{i}, \beta_{i}, a_{i}\right)$. Because solver $i$ does not know other solvers' ability levels, the equilibrium performance $\widetilde{v}^{*}$ of any other solver is uncertain. Let $P_{(j)}^{n}\left[v_{i}, v^{*}\right]$ be the probability that solver $i$ 's performance $v_{i}$ is the $j$-th highest performance when all other $(n-1)$ solvers use performance function $v^{*}$. We compute this probability as

$$
P_{(j)}^{n}\left[v_{i}, v^{*}\right]=\frac{(n-1) !}{(j-1) !(n-j) !} P\left(v_{i}>\widetilde{v}^{*}\right)^{n-j} P\left(v_{i} \leq \widetilde{v}^{*}\right)^{j-1} .
$$

Each solver $i$ is risk-neutral, and maximizes her utility from the contest by solving the following problem:

$$
\max _{e_{i}} \sum_{j=1}^{n} A_{j} P_{(j)}^{n}\left[\beta_{i}+r\left(a_{i} e_{i}\right), v^{*}\right]-c_{i} e_{i}-k=\max _{v_{i}} \sum_{j=1}^{n} A_{j} P_{(j)}^{n}\left[v_{i}, v^{*}\right]-c_{i} r^{-1}\left(v_{i}-\beta_{i}\right) / a_{i}-k .
$$

In equilibrium, $v_{i}=v^{*}\left(c_{i}, \beta_{i}, a_{i}\right)$. Solver $i$ will participate in the contest if her utility is nonnegative; i.e.,

$$
\sum_{j=1}^{n} A_{j} P_{(j)}^{n}\left[v^{*}\left(c_{i}, \beta_{i}, a_{i}\right), v^{*}\right]-c_{i} r^{-1}\left(v^{*}\left(c_{i}, \beta_{i}, a_{i}\right)-\beta_{i}\right) / a_{i}-k \geq 0
$$


We consider the following three interesting and tractable special cases.

Cost-based projects $\left(a_{i}=1, \beta_{i}=0, r\left(e_{i}\right)=\theta e_{i}\right.$ with $\left.\theta>0\right)$ : In a cost-based project that is analyzed by M\&S, solvers are heterogeneous in their cost of effort $c_{i}$, yet they have identical expertise and productivity levels. Solver $i$ with effort $e_{i}$ has performance $v_{i}^{C}\left(e_{i}\right) \equiv \theta e_{i}$. Cost $c_{i}$ is drawn from a continuous distribution $G$ with density $g$ and support $[\underline{c}, \bar{c}] \in \mathbb{R}_{+}$.

Expertise-based projects $\left(a_{i}=1, c_{i}=c, r\left(e_{i}\right)=\theta \log e_{i}\right.$ with $\left.\theta>0\right)$ : In an expertise-based project that is proposed by T\&X, solvers are heterogeneous in their expertise levels, while they are identical in their productivity and cost of effort. Solver $i$ with effort $e_{i}$ has performance $v_{i}^{E}\left(e_{i}, \beta_{i}\right) \equiv$ $\beta_{i}+\theta \log \left(e_{i}\right)$. Expertise level $\beta_{i}$ is drawn from a continuous distribution $F$ with density $f$ and support $[\underline{\beta}, \bar{\beta}] \in \mathbb{R}$.

Productivity-based projects $\left(\beta_{i}=0, c_{i}=c\right)$ : In a productivity-based project, solvers are heterogeneous in their productivity levels, while they are identical in their expertise and cost of effort. Solver $i$ with effort $e_{i}$ has performance $v_{i}^{P}\left(a_{i}, e_{i}\right) \equiv r\left(a_{i} e_{i}\right)$. Productivity level $a_{i}$ is drawn from a continuous distribution $H$ with density $h$ and support $[\underline{a}, \bar{a}] \in \mathbb{R}_{+}$.

Let $\widetilde{\beta}_{(j)}^{n}, F_{(j)}^{n}$, and $f_{(j)}^{n}\left(\right.$ resp., $\widetilde{a}_{(j)}^{n}, H_{(j)}^{n}$, and $\left.h_{(j)}^{n}\right)$ represent the random variable, the distribution function, and the density function of the $j$-th highest expertise (resp., productivity) among $n$ solvers, respectively. Note that $\widetilde{\beta}_{(j)}^{n}\left(\right.$ resp., $\left.\widetilde{a}_{(j)}^{n}\right)$ corresponds to the $(n-j+1)$-st order statistic.

\section{Analysis of Productivity-Based Projects}

We show in Lemma EC.1 of the Online Appendix that cost-based projects of M\&S and expertisebased projects of T\&X can be represented as special cases of productivity-based projects with a general effort function $r$ and a general productivity distribution $H$. Thus, by analyzing productivity-based projects, we can also characterize cost-based and expertise-based projects. We focus on winner-take-all contests, and assume that the fixed cost $k=0$ for simplicity. ${ }^{4}$

\footnotetext{
4 The results can easily be extended to the case with multiple awards and a positive fixed cost. It can be shown that the winner-take-all scheme is optimal when the effort function $r$ is not too concave (e.g., $r(e)=\theta \log (e))$. See also Ales et al.
} (2016b). 
We first derive the solver's equilibrium effort $e^{*}$ and performance $v^{*}$. Suppose that $v^{*}$ is an increasing function (which is verified later). Then, from (2), solver $i$ 's probability of winning the contest is $P_{(1)}^{n}\left[v_{i}, v^{*}\right]=P\left(v_{i}>\widetilde{v}^{*}\right)^{n-1}=H_{(1)}^{n-1}\left(\left(v^{*}\right)^{-1}\left(v_{i}\right)\right)$. Given the winner prize $A$ and other solvers' performance function $v^{*}$, solver $i$ determines her equilibrium performance $v^{*}\left(a_{i}\right)$ by solving the following problem modified from (3):

$$
\max _{v_{i}} A H_{(1)}^{n-1}\left(\left(v^{*}\right)^{-1}\left(v_{i}\right)\right)-c r^{-1}\left(v_{i}\right) / a_{i} .
$$

LEMMA 1. For a general productivity distribution $H$ and a general effort function $r$, a solver with productivity $a_{i}$ has equilibrium effort $e^{*}\left(a_{i}\right)=\frac{A}{c a_{i}} \int_{\underline{a}}^{a_{i}} a h_{(1)}^{n-1}(a) d a$ and performance $v^{*}\left(a_{i}\right)=$ $r\left(\frac{A}{c} \int_{\underline{a}}^{a_{i}} a h_{(1)}^{n-1}(a) d a\right)$.

Next, we discuss how the solver's equilibrium effort changes with the number of her competitors. Let $e^{*, n}$ denote the equilibrium effort when there are $n$ solvers in the contest. Figure 1(a) illustrates that an additional solver has a minimal effect on low-productivity solvers, whereas it has a negative effect on moderate-productivity solvers, and it has a positive effect on high-productivity solvers. In order to identify the factors that drive the pattern of Figure 1(a), we decompose the equilibrium effort $e^{*, n}\left(a_{i}\right)$ as

$$
e^{*, n}\left(a_{i}\right)=\frac{A}{c a_{i}} H_{(1)}^{n-1}\left(a_{i}\right) \int_{\underline{a}}^{a_{i}} a \frac{h_{(1)}^{n-1}(a)}{H_{(1)}^{n-1}(a)} d a=\frac{A}{c a_{i}} H_{(1)}^{n-1}\left(a_{i}\right) \times E\left[\widetilde{a}_{(1)}^{n-1} \mid \widetilde{a}_{(1)}^{n-1}<a_{i}\right],
$$

where $H_{(1)}^{n-1}\left(a_{i}\right)$ represents solver $i$ 's probability of winning the contest which corresponds to her probability of having a higher productivity than all other solvers, and $E\left[\widetilde{a}_{(1)}^{n-1} \mid \widetilde{a}_{(1)}^{n-1}<a_{i}\right]$ represents the expected productivity of the runner-up, given that solver $i$ is the winner.

PROPOSITION 1. For a general productivity distribution $H$ and a general effort function $r, H_{(1)}^{n}\left(a_{i}\right)-$ $H_{(1)}^{n-1}\left(a_{i}\right)<0$ and $E\left[\widetilde{a}_{(1)}^{n} \mid \widetilde{a}_{(1)}^{n}<a_{i}\right]-E\left[\widetilde{a}_{(1)}^{n-1} \mid \widetilde{a}_{(1)}^{n-1}<a_{i}\right]>0$ for all $n$ and $a_{i} \in(\underline{a}, \bar{a})$. Moreover, for any $n$, there exists $\widehat{a}(n) \in[\underline{a}, \bar{a})$ such that for all $a_{i} \in(\widehat{a}(n), \bar{a}), e^{*, n+1}\left(a_{i}\right)>e^{*, n}\left(a_{i}\right)$ and $v^{*, n+1}\left(a_{i}\right)>v^{*, n}\left(a_{i}\right)$.

Proposition 1 shows that a larger number of solvers $n$ reduces solver $i$ 's probability of winning the contest, $H_{(1)}^{n}\left(a_{i}\right)$ (see Figure 1(b)). This negative externality is the intuitive explanation offered by T\&X who argue that every solver will reduce her effort with more solvers in the contest. However, as illustrated in Figure 1(c), the expected productivity of the runner-up given that solver 


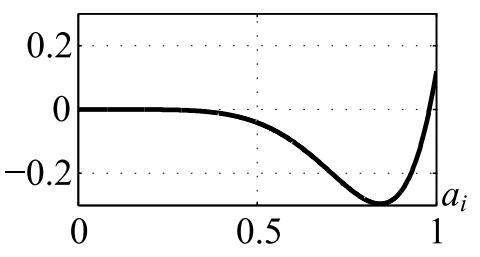

(a) $e^{*, n+1}\left(a_{i}\right)-e^{*, n}\left(a_{i}\right)$

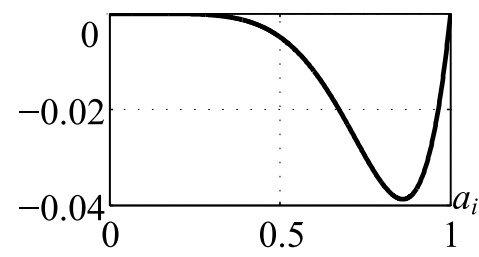

(b) $H_{(1)}^{n}\left(a_{i}\right)-H_{(1)}^{n-1}\left(a_{i}\right)$

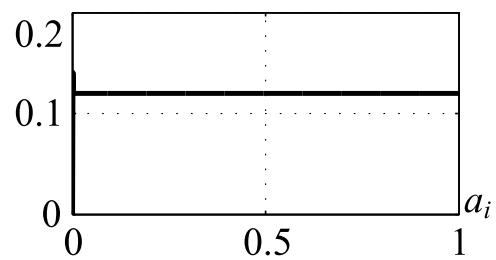

(c) $E\left[\widetilde{a}_{(1)}^{n} \mid \widetilde{a}_{(1)}^{n}<a_{i}\right]-E\left[\widetilde{a}_{(1)}^{n-1} \mid \widetilde{a}_{(1)}^{n-1}<a_{i}\right]$

Figure 1 The impact of an additional solver on (a) effort, (b) solver $i$ 's probability of winning, and (c) the expected productivity of the runner-up given that solver $i$ wins. Setting: Productivity level $a_{i}$ is drawn from Beta distribution with parameters 0.7 and $1, n=10, r(e)=\frac{e^{0.9}}{0.9}, A=1, c=0.1$, and $k=0$.

$i$ is the winner (i.e., $E\left[\widetilde{a}_{(1)}^{n-1} \mid \widetilde{a}_{(1)}^{n-1}<a_{i}\right]$ ) increases with the number of solvers $n$. This driver provides an opposing force to the reduced probability of winning. For high-productivity solvers, the incentives to exert higher effort to win the contest are stronger, and hence their equilibrium effort and performance can increase with an additional participant (see Figure 1(a) for illustration). This result is also empirically observed by Boudreau et al. (2012) for software development contests organized by TopCoder. Finally, Proposition 1 implies that there are high-expertise solvers who would raise their effort with more entrants in an expertise-based project (which is a special case of a productivity-based project). This conflicts with the result of $T \& X$, and it signals a problem in their analysis.

\section{Analysis of Expertise-Based Projects}

In this section, we first describe the analysis of $T \& X$ briefly. Then we derive the correct equilibrium effort $e^{*}$, and discuss managerial implications of this correction. T\&X consider a contest in which the seeker offers two prizes, $A_{1}$ to the winner and $A_{2}$ to the runner-up with $A_{1} \geq A_{2}$. Suppose that the performance of all solvers except solver $i$ is based on the best-response performance function $v^{*}\left(\beta_{i}\right)$, which is continuously differentiable and increasing in the expertise level $\beta_{i}$. Given that all other solvers' performance function is $v^{*}$, each participant $i$ solves the following problem modified from (3):

$$
\max _{v_{i}} A_{1} F_{(1)}^{n-1}\left(\left(v^{*}\right)^{-1}\left(v_{i}\right)\right)+A_{2}(n-1)\left\{F_{(1)}^{n-2}\left(\left(v^{*}\right)^{-1}\left(v_{i}\right)\right)-F_{(1)}^{n-1}\left(\left(v^{*}\right)^{-1}\left(v_{i}\right)\right)\right\}-c r^{-1}\left(v_{i}-\beta_{i}\right)-k
$$




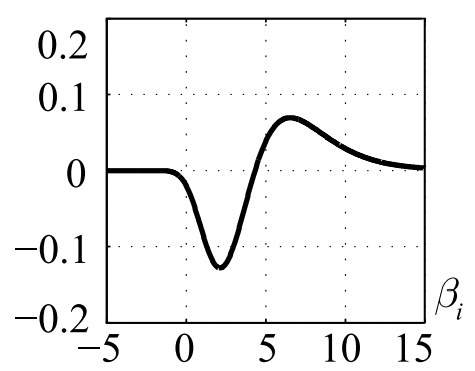

(a) $e^{*, n+1}\left(\beta_{i}\right)-e^{*, n}\left(\beta_{i}\right)$.

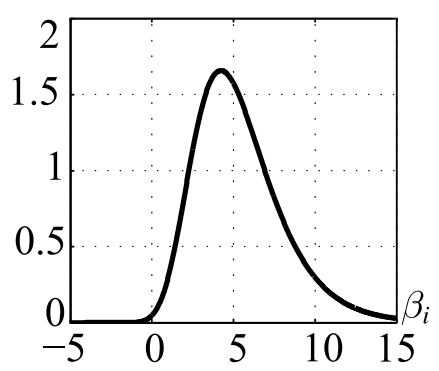

(b) The effort $e^{*}\left(\beta_{i}\right)$.

Figure 2 The true equilibrium effort $e^{*}$ in an expertise-based project when $\beta_{i}$ is drawn from a Gumbel distribution with mean 0 and scale parameter $\lambda=2, n=10, A=\theta=1$, and $c=0.1$. The same parameter values are used in Figure 2 of T\&X.

T\&X solve the differential equation in the first-order condition of (7) by using the Fourier method under the boundary condition $v^{*}(\underline{\beta})=\underline{\beta}$ to obtain the effort

$$
e^{T}\left(\beta_{i}\right) \equiv r^{-1}\left(v^{*}\left(\beta_{i}\right)-\beta_{i}\right)=\frac{A_{1} F\left(\beta_{i}\right)^{n-1}+A_{2}(n-1)\left[F\left(\beta_{i}\right)^{n-2}-F\left(\beta_{i}\right)^{n-1}\right]-k}{c} .
$$

LEMMA 2. The effort $e^{T}$ given in (8) (which is copied from equation (3) in the e-companion of Terwiesch and $X u$ (2008)) cannot be an equilibrium effort.

As we detail in the proof of Lemma $2, e^{T}$ in (8) cannot be an equilibrium effort because: (i) Although T\&X assume a best-response effort function $e^{T}\left(\beta_{i}\right)$ is increasing in $\beta_{i}$, this is not necessarily true as we demonstrate later; (ii) the boundary condition $v^{*}(\underline{\beta})=\underline{\beta}$ does not always hold; and (iii) the differential equation in the first-order condition of (7) does not satisfy the necessary conditions for the Fourier method. Equation (17) in the Appendix provides the correct version of (8), which is obtained by solving the first-order condition of (7) directly (without the Fourier method) using the boundary condition of $e^{*}(\underline{\beta})=0$.

We next present the revised version of Theorem 1B in T\&X that concerns expertise-based projects. As in Theorem 1B, we consider the winner-take-all scheme with $A_{1}=A$ and $A_{2}=0$.

PROPOSITION 2. In an expertise-based project, only solvers with expertise higher than $\beta^{f}=$ $F^{-1}\left(\left(k / A^{*}\right)^{1 /(n-1)}\right)$ will participate, where $A^{*}$ is the optimal winner prize. The effort of a participating 


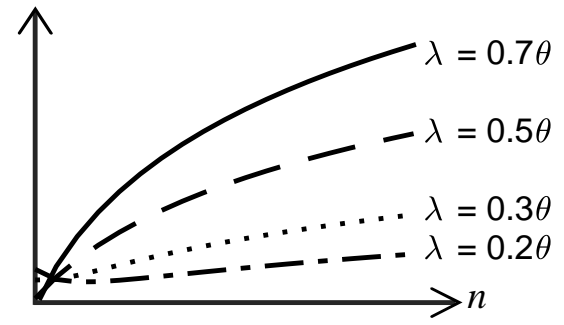

(a) The seeker's true profit $\Pi$.

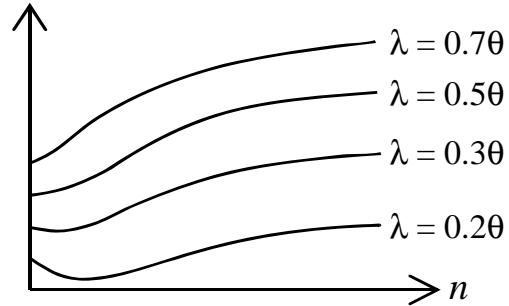

(b) The seeker's profit $\Pi$ in Figure 2(g) of T\&X.

Figure 3 The comparison of the seeker's true profit with that of T\&X for an expertise-based project when $\beta_{i}$ is drawn from a Gumbel distribution with mean 0 and scale parameter $\lambda, \rho=1, A=\theta=1$, and $c=0.1$.

solver with expertise $\beta_{i} \in\left[\beta^{f}, \bar{\beta}\right]$ is $e^{*}\left(\beta_{i}\right)=\frac{1}{c} \int_{\beta f}^{\beta_{i}} \exp \left\{\frac{\beta-\beta_{i}}{\theta}\right\} A^{*} f_{(1)}^{n-1}(\beta) d \beta$. The expected number of participating solvers in an open contest is $n^{*}=n\left(1-\left(k / A^{*}\right)^{1 /(n-1)}\right)$. If $k=0$, the optimal prize $A^{*}=\theta$ and the seeker's profit $\Pi=\int_{\underline{\beta}}^{\bar{\beta}} \theta \log \left(\int_{\underline{\beta}}^{\beta_{i}} \exp \{\beta / \theta\} \frac{\theta}{c} f_{(1)}^{n-1}(\beta) d \beta\right)\left[\rho f_{(1)}^{n}\left(\beta_{i}\right)+(1-\rho) f\left(\beta_{i}\right)\right] d \beta_{i}$.

We now discuss the impact of the incorrectly derived effort $e^{T}$ in T\&X on their Theorems 1A, 1B, and $1 \mathrm{C}$ that concern expertise-based projects. (These theorems also contain results of two other types of contests.) Theorem 1A states that the winner-take-all scheme "may or may not be optimal," and this statement holds under the true equilibrium effort $e^{*}$. Theorem 1B presents the following effort $e^{T}$ :

$$
e^{T}\left(\beta_{i}\right)=\frac{A^{*} F\left(\beta_{i}\right)^{n-1}-k}{c}
$$

The discrepancy between $e^{T}\left(\beta_{i}\right)$ in (9) and $e^{*}\left(\beta_{i}\right)$ in Proposition 2 bears the following implications.

First, although $e^{T}$ in (9) is decreasing in $n$, the true equilibrium effort $e^{*}$ is not always decreasing in $n$; see Figure 2(a) and the earlier discussion in $\S 3$.

Second, although $e^{T}\left(\beta_{i}\right)$ in (9) is increasing in $\beta_{i}$, the true equilibrium effort $e^{*}\left(\beta_{i}\right)$ is not necessarily increasing in expertise level $\beta_{i}$. For example, when $\beta_{i}$ follows a Gumbel distribution as in T\&X, Figure 2(b) shows that the effort $e^{*}\left(\beta_{i}\right)$ decreases with $\beta_{i}$ when $\beta_{i}$ is high (e.g., $\left.\beta_{i}>5\right)$. The intuition from this result is as follows: If a solver knows that she has a relatively higher expertise than most other solvers, then she may not exert as high effort in equilibrium as others do by anticipating that she can still win the contest. 


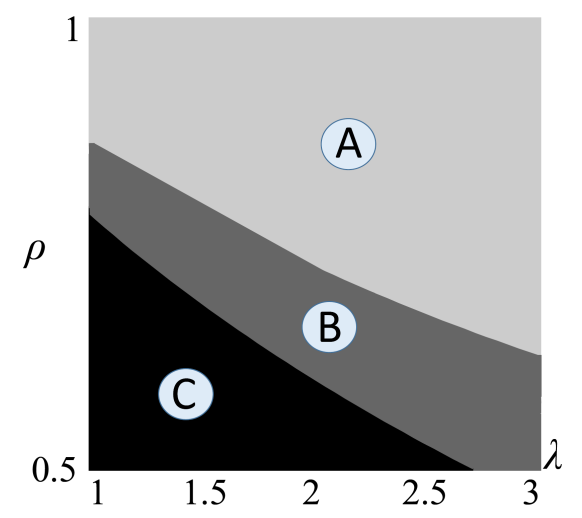

(a) $F \sim$ Gumbel.

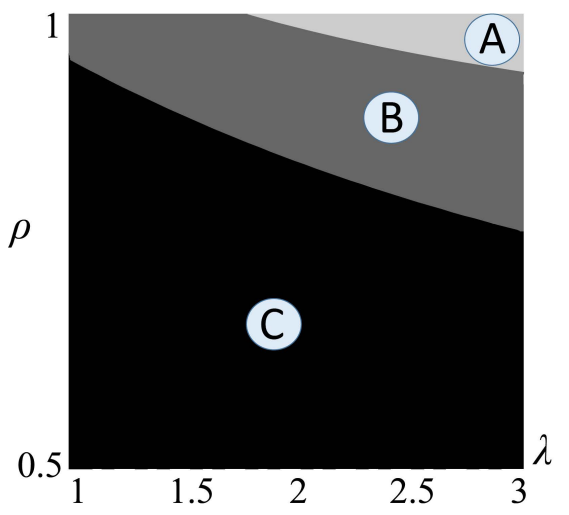

(b) $F \sim$ Weibull.

Figure 4 A set of parameters $(\lambda, \rho)$ under which an open contest is optimal, where region A indicates that an open contest is optimal under both $e^{*}$ and $e^{T}$, region B indicates that an open contest is optimal under $e^{*}$ but not under $e^{T}$, and region $C$ indicates that an open contest is optimal under neither $e^{*}$ nor $e^{T}:$ (a) $F \backsim$ Gumbel with mean 0 and scale parameter $\lambda$ and (b) $F \backsim$ Weibull with mean 0 , shape parameter 1 , and scale parameter $\lambda$. Other parameters: $A=\theta=1$ and $c=0.1$.

Third, as a result of the difference between $e^{T}\left(\beta_{i}\right)$ and $e^{*}\left(\beta_{i}\right)$, the seeker's expected profit $\Pi$ given in Proposition 2 is different from that of T\&X; see Figure 3 for illustration. Consequently, unlike their Theorem 1C, when the expertise distribution $F$ follows a Gumbel distribution with scale parameter $\lambda$ and the seeker is interested in only the best solution (i.e., $\rho=1$ ), even if $\lambda<$ $\theta / 2$, the seeker's profit $\Pi$ can be increasing in $n$, thereby making an open contest optimal. For example, Figure 3(a) displays that $\Pi$ is increasing in $n$ when $\lambda=0.3 \theta$, although $\Pi$ in $\mathrm{T} \& \mathrm{X}$ is not. Furthermore, Figure 4 demonstrates that for a large set of $(\lambda, \rho)$ pairs, an open contest is optimal under the true equilibrium effort $e^{*}\left(\beta_{i}\right)$ but not optimal under $e^{T}\left(\beta_{i}\right)$ in (9); see region B of Figure 4(a)-(b). Interestingly, as Figure 4(b) depicts, when the seeker's weight on the best solution $\rho=$ 1 and the expertise distribution $F$ follows a Weibull distribution, an open contest may not be optimal under $e^{T}\left(\beta_{i}\right)$ although it is always optimal under $e^{*}\left(\beta_{i}\right)$. In addition, whereas an open contest is never optimal when $\rho=0$ under $e^{T}\left(\beta_{i}\right)$ in (9), we can show that an open contest can be optimal under $e^{*}\left(\beta_{i}\right)$. This is because $e^{T}\left(\beta_{i}\right)$ in (9) decreases with the number of solvers $n$ for any expertise level $\beta_{i}$ so the average performance of solvers (i.e., $\left.\int_{\underline{\beta}}^{\bar{\beta}}\left(\beta_{i}+r\left(e^{T}\left(\beta_{i}\right)\right)\right) f\left(\beta_{i}\right) d \beta_{i}\right)$ decreases with $n$; whereas the true equilibrium effort $e^{*}\left(\beta_{i}\right)$ increases with $n$ for high-expertise solvers, and 
hence the true average performance of solvers can increase with $n$. Taken in sum, an open contest is more likely to be optimal than what T\&X asserted because of the positive incentive effect of increased competition on high-expertise solvers. This result helps explain the widespread use of open contests in practice.

\section{Appendix}

Proof of Lemma 1. Suppose that all solvers except solver $i$ have performance based on the best-response performance function $v^{*}\left(a_{i}\right)$, which is assumed to be continuously differentiable and increasing in the productivity $a_{i}$. We can write the best-response effort as $e^{*}\left(a_{i}\right)=$ $r^{-1}\left(v^{*}\left(a_{i}\right)\right) / a_{i}$. Thus, solver $i$ with productivity $a_{i}$ will solve (5) to determine her performance $v_{i}$. Evaluating the first-order condition of (5) at $v_{i}=v^{*}\left(a_{i}\right)=r\left(a_{i} e^{*}\left(a_{i}\right)\right)$, and multiplying it with $a_{i} r^{\prime}\left(a_{i} e^{*}\left(a_{i}\right)\right)\left[a_{i}\left(e^{*}\right)^{\prime}\left(a_{i}\right)+e^{*}\left(a_{i}\right)\right] / c$, we obtain

$$
\frac{a_{i}}{c} A h_{(1)}^{n-1}\left(a_{i}\right)-\left[a_{i}\left(e^{*}\right)^{\prime}\left(a_{i}\right)+e^{*}\left(a_{i}\right)\right]=0 .
$$

Since $v^{*}\left(a_{i}\right)$ is increasing, the least productive solver has no chance of winning $A$, so she will exert zero effort (i.e., $e^{*}(\underline{a})=0$ ). Thus, solving (10) yields the following equilibrium effort and performance:

$$
e^{*}\left(a_{i}\right)=\frac{1}{a_{i}} \int_{\underline{a}}^{a_{i}} \frac{a}{c} A h_{(1)}^{n-1}(a) d a \text { and } v^{*}\left(a_{i}\right)=r\left(\int_{\underline{a}}^{a_{i}} \frac{a}{c} A h_{(1)}^{n-1}(a) d a\right) .
$$

Finally, we verify that the equilibrium performance $v^{*}\left(a_{i}\right)$ is continuously differentiable and increasing in $a_{i}$. Since all of the terms inside $v^{*}\left(a_{i}\right)$ above are continuously differentiable in $a_{i}$, so is $v^{*}$. Thus, $v^{*}\left(a_{i}\right)$ is increasing in $a_{i}$ because $\left(v^{*}\right)^{\prime}\left(a_{i}\right)=r^{\prime}\left(\int_{\underline{a}}^{a_{i}} \frac{a}{c} A h_{(1)}^{n-1}(a) d a\right) \times \frac{a_{i}}{c} A h_{(1)}^{n-1}\left(a_{i}\right)>0$.

Proof of Proposition 1. For any $a_{i}<\bar{a}$, we have $H_{(1)}^{n}\left(a_{i}\right)-H_{(1)}^{n-1}\left(a_{i}\right)=\left(H\left(a_{i}\right)-1\right) H\left(a_{i}\right)^{n-1}<0$. Furthermore, by Corollary 1.C.38 and Theorem 1.C.5 of Shaked and Shanthikumar (2007), $\widetilde{a}_{(1)}^{n}$ dominates $\widetilde{a}_{(1)}^{n-1}$ in the likelihood ratio order, and $\left[\widetilde{a}_{(1)}^{n} \mid \widetilde{a}_{(1)}^{n}<a_{i}\right]$ first-order stochastically dominates $\left[\widetilde{a}_{(1)}^{n-1} \mid \widetilde{a}_{(1)}^{n-1}<\right.$ $\left.a_{i}\right]$ (and the reverse is not true for $a_{i}>\underline{a}$ ). Then, for any $a_{i}>\underline{a}, E\left[\widetilde{a}_{(1)}^{n} \mid \widetilde{a}_{(1)}^{n}<a_{i}\right]-E\left[\widetilde{a}_{(1)}^{n-1} \mid \widetilde{a}_{(1)}^{n-1}<\right.$ $\left.a_{i}\right]>0$. For the solver with productivity $\bar{a}$, the equilibrium effort is $e^{*, n}(\bar{a})=\frac{1}{\bar{a}} \int_{\underline{a}}^{\bar{a}} \frac{A^{*} a}{c} h_{(1)}^{n-1}(a) d a=$ $\frac{A^{*}}{c \bar{a}} E\left[\widetilde{a}_{(1)}^{n-1}\right]$. From above, $\delta \equiv E\left[\widetilde{a}_{(1)}^{n}\right]-E\left[\widetilde{a}_{(1)}^{n-1}\right]=E\left[\widetilde{a}_{(1)}^{n} \mid \widetilde{a}_{(1)}^{n}<\bar{a}\right]-E\left[\widetilde{a}_{(1)}^{n-1} \mid \widetilde{a}_{(1)}^{n-1}<\bar{a}\right]>0$. For any $a_{i}$, we have

$e^{*, n+1}\left(a_{i}\right)-e^{*, n}\left(a_{i}\right)=\frac{A^{*}}{c a_{i}} \int_{\underline{a}}^{a_{i}} a\left[h_{(1)}^{n}(a)-h_{(1)}^{n-1}(a)\right] d a=\frac{A^{*}}{c a_{i}}\left(E\left[\widetilde{a}_{(1)}^{n}\right]-E\left[\widetilde{a}_{(1)}^{n-1}\right]-\int_{a_{i}}^{\bar{a}} a\left[h_{(1)}^{n}(a)-h_{(1)}^{n-1}(a)\right] d a\right)$.

As $a_{i}$ approaches $\bar{a}$, the term $\int_{a_{i}}^{\bar{a}} a\left[h_{(1)}^{n}(a)-h_{(1)}^{n-1}(a)\right] d a$ approaches 0 , so there exists $\widehat{a}^{1} \in[\underline{a}, \bar{a})$ such that for $a_{i}>\widehat{a}^{1}$, we have $\int_{a_{i}}^{\bar{a}} a\left[h_{(1)}^{n}(a)-h_{(1)}^{n-1}(a)\right]<\delta$. Then, for any $a_{i} \in\left(\widehat{a}^{1}, \bar{a}\right)$, we have 


$$
e^{*, n+1}\left(a_{i}\right)-e^{*, n}\left(a_{i}\right)=\frac{A^{*}}{c a_{i}}\left(\delta-\int_{a_{i}}^{\bar{a}} a\left[h_{(1)}^{n}(a)-h_{(1)}^{n-1}(a)\right] d a\right)>0 .
$$

Similarly, noting that the effort function $r$ is increasing, the equilibrium performance $v^{*}$ satisfies

$$
v^{*, n+1}(\bar{a})=r\left(\frac{A^{*}}{c} E\left[\widetilde{a}_{(1)}^{n}\right]\right)>r\left(\frac{A^{*}}{c} E\left[\widetilde{a}_{(1)}^{n-1}\right]\right)=v^{*, n}(\bar{a}) .
$$

$v^{*, n+1}\left(a_{i}\right)-v^{*, n}\left(a_{i}\right)$ is continuous because $v^{*, n}\left(a_{i}\right)$ is continuous. Thus, there exists $\widehat{a}^{2} \in[\underline{a}, \bar{a})$ such that $v^{*, n+1}\left(a_{i}\right)-v^{*, n}\left(a_{i}\right)>0$ for all $a_{i} \in\left(\widehat{a}^{2}, \bar{a}\right)$; so letting $\widehat{a}(n)=\max \left\{\widehat{a}^{1}, \widehat{a}^{2}\right\}$ yields the proposed result.

Proof of Lemma 2. To prove that $e^{T}$ cannot be an equilibrium effort, we describe how Terwiesch and $\mathrm{Xu}(2008)$ (hereinafter $\mathrm{T} \& \mathrm{X}$ ) derive $e^{T}$, and point out the problems in their derivation. To derive the equilibrium effort of a solver, T\&X take the perspective of solver $i$ with expertise level $\beta_{i}$, and assume that all other solvers exert effort based on the best-response function $e^{T}\left(\beta_{i}\right)$, which is continuously differentiable and increasing in $\beta_{i}{ }^{5}$ They also let $v^{T}\left(\beta_{i}\right)=\beta_{i}+r\left(e^{T}\left(\beta_{i}\right)\right)$ be the best-response performance of solvers, and assume that the fixed cost $k=0$. With a performance $v_{i}$, the probability that solver $i$ will receive $A_{1}$ is $P\left\{v_{i} \geq v^{T}(\widetilde{\beta})\right\}^{n-1}=F\left(\left(v^{T}\right)^{-1}\left(v_{i}\right)\right)^{n-1}$, and the probability that she will receive $A_{2}$ is $(n-1) P\left\{v_{i}<v^{T}(\widetilde{\beta})\right\} P\left\{v_{i} \geq v^{T}(\widetilde{\beta})\right\}^{n-2}=(n-1)(1-$ $\left.F\left(\left(v^{T}\right)^{-1}\left(v_{i}\right)\right)\right) F\left(\left(v^{T}\right)^{-1}\left(v_{i}\right)\right)^{n-2}$. Then, solver $i$ solves the following problem given that all other solvers' performance function is $v^{T}\left(\beta_{i}\right)$ :

$$
\max _{v_{i}} A_{1} F_{(1)}^{n-1}\left(\left(v^{T}\right)^{-1}\left(v_{i}\right)\right)+A_{2}(n-1)\left\{F_{(1)}^{n-2}\left(\left(v^{T}\right)^{-1}\left(v_{i}\right)\right)-F_{(1)}^{n-1}\left(\left(v^{T}\right)^{-1}\left(v_{i}\right)\right)\right\}-c r^{-1}\left(v_{i}-\beta_{i}\right) .
$$

The first-order condition evaluated at $v_{i}=v^{T}\left(\beta_{i}\right)=\beta_{i}+r\left(e^{T}\left(\beta_{i}\right)\right)$, after simplifications, yields:

$$
\left(v^{T}\right)^{\prime}\left(\beta_{i}\right) c=r^{\prime}\left(r^{-1}\left(v^{T}\left(\beta_{i}\right)-\beta_{i}\right)\right)\left[A_{1} f_{(1)}^{n-1}\left(\beta_{i}\right)+A_{2}(n-1)\left(f_{(1)}^{n-2}\left(\beta_{i}\right)-f_{(1)}^{n-1}\left(\beta_{i}\right)\right)\right] .
$$

T\&X solve (12) using the Fourier method under the boundary condition $v^{T}(\underline{\beta})=\underline{\beta}$ to obtain the equilibrium effort $e^{T}$ of a solver with expertise $\beta_{i}$ in (8).

There are three reasons why $e^{T}$ in (8) cannot be an equilibrium effort. First, (12) does not satisfy the necessary conditions for the Fourier method, and hence (8) does not solve (12). To show this, we substitute $\left(v^{T}\right)^{\prime}\left(\beta_{i}\right)=1+r^{\prime}\left(e^{T}\left(\beta_{i}\right)\right)\left(e^{T}\right)^{\prime}\left(\beta_{i}\right)$ and $\left(e^{T}\right)^{\prime}\left(\beta_{i}\right)$ from (8) (where $F\left(\beta_{i}\right)^{n-1}=F_{(1)}^{n-1}\left(\beta_{i}\right)$ ) into the left hand side of (12), and show that it is not equal to the right hand side of (12):

$$
\left(v^{T}\right)^{\prime}\left(\beta_{i}\right) c=c+r^{\prime}\left(r^{-1}\left(v^{T}\left(\beta_{i}\right)-\beta_{i}\right)\right)\left[A_{1} f_{(1)}^{n-1}\left(\beta_{i}\right)+A_{2}(n-1)\left(f_{(1)}^{n-2}\left(\beta_{i}\right)-f_{(1)}^{n-1}\left(\beta_{i}\right)\right)\right]
$$

${ }^{5}$ Note that we use a best-response performance function $v^{*}\left(\beta_{i}\right)$, whereas T\&X use a best-response effort function $e^{T}\left(\beta_{i}\right)$. Increasing $e^{T}\left(\beta_{i}\right)$ implies increasing $v^{*}\left(\beta_{i}\right)$, but not vice versa due to the term $\beta_{i}$ in $v^{*}\left(\beta_{i}\right)=\beta_{i}+r\left(e^{T}\left(\beta_{i}\right)\right)$. 


$$
\neq r^{\prime}\left(r^{-1}\left(v^{T}\left(\beta_{i}\right)-\beta_{i}\right)\right)\left[A_{1} f_{(1)}^{n-1}\left(\beta_{i}\right)+A_{2}(n-1)\left(f_{(1)}^{n-2}\left(\beta_{i}\right)-f_{(1)}^{n-1}\left(\beta_{i}\right)\right)\right]
$$

because the unit cost of effort $c$ is positive. Second, T\&X use the boundary condition of $v^{T}(\underline{\beta})=\underline{\beta}$, which does not always hold. For instance, the effort function $r(e)=\theta \log (e)$ used in Theorems 1B and $1 C$ yields $\lim _{\beta_{i} \rightarrow \underline{\beta}} v^{T}\left(\beta_{i}\right)=\lim _{\beta_{i} \rightarrow \underline{\beta}}\left(\beta_{i}+\theta \log e^{T}\left(\beta_{i}\right)\right)=-\infty$. Thus, when $\underline{\beta} \neq-\infty$, this boundary condition is invalid (e.g., $F$ follows a beta or gamma distribution). Third, they start the proof by assuming that $e^{T}\left(\beta_{i}\right)$ is increasing with $\beta_{i}$, but the equilibrium effort is not necessarily increasing with $\beta_{i}$. Therefore, the solver's effort $e^{T}$ in (8) is different from the true equilibrium effort $e^{*}$.

Proof of Proposition 2. The first-order condition of (7) evaluated at $v_{i}=v^{*}\left(\beta_{i}\right)$ yields:

$$
\left(v^{*}\right)^{\prime}\left(\beta_{i}\right) c=r^{\prime}\left(r^{-1}\left(v^{*}\left(\beta_{i}\right)-\beta_{i}\right)\right)\left[A_{1} f_{(1)}^{n-1}\left(\beta_{i}\right)+A_{2}(n-1)\left(f_{(1)}^{n-2}\left(\beta_{i}\right)-f_{(1)}^{n-1}\left(\beta_{i}\right)\right)\right] .
$$

Letting the equilibrium effort $e^{*}\left(\beta_{i}\right)=r^{-1}\left(v^{*}\left(\beta_{i}\right)-\beta_{i}\right)$, we have $r^{\prime}\left(r^{-1}\left(v^{*}\left(\beta_{i}\right)-\beta_{i}\right)\right)=\theta / e^{*}\left(\beta_{i}\right)$ and $\left(v^{*}\right)^{\prime}\left(\beta_{i}\right)=1+r^{\prime}\left(e^{*}\left(\beta_{i}\right)\right)\left(e^{*}\right)^{\prime}\left(\beta_{i}\right)=1+\theta\left(e^{*}\right)^{\prime}\left(\beta_{i}\right) / e^{*}\left(\beta_{i}\right)$. Substituting these into (13), and by multiplying both sides of (13) with $\frac{e^{*}\left(\beta_{i}\right)}{c \theta} \exp \left\{\frac{\beta_{i}}{\theta}\right\}$, we obtain the following equation that $e^{*}\left(\beta_{i}\right)$ satisfies:

$$
\exp \left\{\frac{\beta_{i}}{\theta}\right\} \frac{e^{*}\left(\beta_{i}\right)}{\theta}+\exp \left\{\frac{\beta_{i}}{\theta}\right\}\left(e^{*}\right)^{\prime}\left(\beta_{i}\right)=\exp \left\{\frac{\beta_{i}}{\theta}\right\}\left[\frac{A_{1}}{c} f_{(1)}^{n-1}\left(\beta_{i}\right)+\frac{A_{2}(n-1)}{c}\left(f_{(1)}^{n-2}\left(\beta_{i}\right)-f_{(1)}^{n-1}\left(\beta_{i}\right)\right)\right] .
$$

Note that the left hand side of (14) is the derivative of $\exp \left\{\beta_{i} / \theta\right\} e^{*}\left(\beta_{i}\right)$ with respect to $\beta_{i}$. When fixed $\operatorname{cost} k=0$, because a solver with expertise level $\underline{\beta}$ has no chance of winning the contest, her effort $e^{*}(\underline{\beta})=0$. Thus, by integrating both sides of (14) and then multiplying both sides with $\exp \left\{-\beta_{i} / \theta\right\}$, we obtain

$$
e^{*}\left(\beta_{i}\right)=\frac{1}{c} \int_{\underline{\beta}}^{\beta_{i}} \exp \left\{\frac{\beta-\beta_{i}}{\theta}\right\}\left[A_{1} f_{(1)}^{n-1}(\beta)+A_{2}(n-1)\left(f_{(1)}^{n-2}(\beta)-f_{(1)}^{n-1}(\beta)\right)\right] d \beta .
$$

Then $v^{*}\left(\beta_{i}\right)=\theta \log \left(\int_{\underline{\beta}}^{\beta_{i}} \exp \left\{\frac{\beta}{\theta}\right\}\left[\frac{A_{1}}{c} f_{(1)}^{n-1}(\beta)+\frac{A_{2}}{c}(n-1)\left(f_{(1)}^{n-2}(\beta)-f_{(1)}^{n-1}(\beta)\right)\right] d \beta\right)$. Since all of the terms of $v^{*}\left(\beta_{i}\right)$ are continuously differentiable, so is $v^{*}\left(\beta_{i}\right)$. Also, $v^{*}\left(\beta_{i}\right)$ is increasing because

$$
\left(v^{*}\right)^{\prime}\left(\beta_{i}\right)=\left(\theta /\left(c e^{*}\left(\beta_{i}\right)\right)\right) \exp \left\{\beta_{i} / \theta\right\}\left[A_{1} f_{(1)}^{n-1}\left(\beta_{i}\right)+A_{2}(n-1)\left(f_{(1)}^{n-2}\left(\beta_{i}\right)-f_{(1)}^{n-1}\left(\beta_{i}\right)\right)\right]>0 .
$$

Next, when there is a fixed cost $k \geq 0$, only solvers with expertise level $\beta_{i} \in\left[\beta^{f}, \bar{\beta}\right]$ will participate in the contest, where $\beta^{f}$ satisfies $e^{*}\left(\beta^{f}\right)=0$ and the zero-utility condition (see (4)):

$$
A_{1} F_{(1)}^{n-1}\left(\beta^{f}\right)+A_{2}(n-1)\left[F_{(1)}^{n-2}\left(\beta^{f}\right)-F_{(1)}^{n-1}\left(\beta^{f}\right)\right]-k=0 .
$$

Solving the first-order condition with $e^{*}\left(\beta^{f}\right)=0$, we obtain the following correct version of (8):

$$
e^{*}\left(\beta_{i}\right)=\frac{1}{c} \int_{\beta^{f}}^{\beta_{i}} \exp \left\{\frac{\beta-\beta_{i}}{\theta}\right\}\left[A_{1} f_{(1)}^{n-1}(\beta)+A_{2}(n-1)\left(f_{(1)}^{n-2}(\beta)-f_{(1)}^{n-1}(\beta)\right)\right] d \beta .
$$


For $k \geq 0$, from (16) with $A_{1}=A^{*}$ and $A_{2}=0$, we obtain $\beta^{f}=F^{-1}\left(\left(k / A^{*}\right)^{1 /(n-1)}\right)$. For any solver with expertise $\beta_{i}<\beta^{f}$, constraint (4) is violated, so only solvers with $\beta_{i} \geq \beta^{f}$ will participate. Effort $e^{*}$ is obtained by substituting $A_{1}=A^{*}$ and $A_{2}=0$ into (17). The probability that a solver participates is $P\left(\widetilde{\beta} \geq \beta^{f}\right)=1-\left(k / A^{*}\right)^{1 /(n-1)}$, so the expected number of participating solvers in an open contest is $n^{*}=n\left(1-\left(k / A^{*}\right)^{1 /(n-1)}\right)$. If $k=0$, then $\beta^{f}=F^{-1}(0)=\underline{\beta}$. Thus, from (1), the seeker's profit

$$
\Pi=\int_{\underline{\beta}}^{\bar{\beta}} \theta \log \left(\int_{\underline{\beta}}^{\beta_{i}} \exp \{\beta / \theta\} \frac{A^{*}}{c} f_{(1)}^{n-1}(\beta) d \beta\right)\left[\rho f_{(1)}^{n}\left(\beta_{i}\right)+(1-\rho) f\left(\beta_{i}\right)\right] d \beta_{i}-A^{*} .
$$

The optimal prize satisfies the first-order condition $\int_{\underline{\beta}}^{\bar{\beta}} \theta \frac{1}{A^{*}}\left[\rho f_{(1)}^{n}\left(\beta_{i}\right)+(1-\rho) f\left(\beta_{i}\right)\right] d \beta_{i}-1=\theta \frac{1}{A^{*}}-$ $1=0$. Thus, the optimal prize $A^{*}=\theta$, and the expected profit is obtained by plugging $A^{*}=\theta$ in (18).

\section{Acknowledgments}

We appreciate helpful comments from seminar participants at Carnegie Mellon University, Cornell University, Stanford University, M\&SOM Annual Conference, The Annual Conference of the Production and Operations Management Society, INFORMS International Conference, and INFORMS Annual Meeting. We also thank the department editor Gad Allon, his review team, and Yi Xu for their guidance and constructive comments. This work is partially supported by the Carnegie Bosch Research Grant, Carnegie Mellon University.

\section{References}

Ales, L., S. Cho, E. Korpeoglu. 2016a. Innovation tournaments with multiple contributors. Working Paper, Carnegie Mellon University.

Ales, L., S. Cho., E. Korpeoglu. 2016b. Optimal award scheme in innovation tournaments. To appear in Oper. Res.

Boudreau, K. J., C. E. Helfat, K. R. Lakhani, M. Menietti. 2012. Field evidence on individual behavior and performance in rank-order tournaments. Working Paper, Harvard Business School .

Fullerton, R. L., R. P. McAfee. 1999. Auctioning entry into tournaments. J. Polit. Econ. 107(3) 573-605.

Hopkins, E., T. Kornienko. 2007. Cross and double cross: Comparative statics in first price and all pay auctions. The B.E J. Theor. Econ. 7(1). Article 19.

Moldovanu, B., A. Sela. 2006. Contest architecture. J. Econ. Theory 126 70-96.

Shaked, M., J. G. Shanthikumar. 2007. Stochastic Orders. Springer, New York. 
Taylor, C. R. 1995. Digging for golden carrots: an analysis of research tournaments. Amer. Econ. Rev. 85(4) 872-890.

Terwiesch, C., Y. Xu. 2008. Innovation contests, open innovation and multiagent problem solving. Manage. Sci. 54(9) 1529-1543. 


\title{
E-Companion to "Incentives in Contests with Heterogeneous Solvers"
}

\author{
Ersin Körpeoğlu · Soo-Haeng Cho \\ School of Management, University College London, London, UK E14 5AB \\ Tepper School of Business, Carnegie Mellon University, Pittsburgh, PA 15213 \\ e.korpeoglu@ucl.ac.uk·soohaeng@andrew.cmu.edu
}

LEMMA EC.1. (a) When the effort function $r\left(e_{i}\right)=\theta e_{i}$ where $\theta>0$, the equilibrium performance satisfies $v^{*, P}\left(a_{i}\right)=v^{*, C}\left(c /\left(\theta a_{i}\right)\right)$ for any $a_{i} \in[\underline{a}, \bar{a}]$. Thus, a cost-based project in which the cost level $c_{i}$ is drawn from a general distribution $G$ is a productivity-based project in which productivity level $a_{i}=c /\left(\theta c_{i}\right)$ is drawn from distribution $H\left(a_{i}\right)=1-G\left(c /\left(\theta a_{i}\right)\right)$ and the unit cost of effort is $c$.

(b) When the effort function $r\left(e_{i}\right)=\theta \log e_{i}$ where $\theta>0$, the equilibrium performance satisfies $v^{*, P}\left(a_{i}\right)=$ $v^{*, E}\left(\theta \log \left(a_{i}\right)\right)$ for any $a_{i} \in[\underline{a}, \bar{a}]$. Thus, an expertise-based project in which the expertise level $\beta_{i}$ is drawn from a general distribution $F$ is a productivity-based project in which productivity level $a_{i}=\exp \left(\beta_{i} / \theta\right)$ is drawn from distribution $H\left(a_{i}\right)=F\left(\theta \log \left(a_{i}\right)\right)$.

Proof. In a productivity-based project, from solver $i$ 's perspective, another solver's performance is a random variable $\widetilde{v}^{*, P} \equiv v^{*, P}(\widetilde{a})$. Thus, plugging $P_{(j)}^{n}\left[v_{i}, v^{*}\right]$ expression of (2) into (3), and simplifying it for productivity-based projects, we obtain solver $i$ 's problem as follows:

$$
\max _{v_{i}} \sum_{j=1}^{n} A_{j} \frac{(n-1) !}{(j-1) !(n-j) !} P\left(v_{i}>\widetilde{v}^{*, P}\right)^{n-j} P\left(v_{i} \leq \widetilde{v}^{*, P}\right)^{j-1}-c \frac{r^{-1}\left(v_{i}\right)}{a_{i}}-k .
$$

(a) In a cost-based project, all solvers except solver $i$ have performance based on $v^{*, C}\left(c_{i}\right)$. We will construct a bijective mapping $\eta: R_{+} \rightarrow R_{+}$from a solver's cost $c_{i}$ to a productivity $a_{i}$ (i.e., $\eta\left(c_{i}\right)=$ $\left.a_{i}\right)$ such that given that all other solvers have performance $v^{*, P}\left(a_{i}\right)=v^{*, C}\left(c_{i}\right)$, solver $i$ will have the same best-response performance. Define solver $i$ 's productivity as $a_{i}=\eta\left(c_{i}\right)=c /\left(\theta c_{i}\right)$. Given $v^{*, P}\left(a_{i}\right)=v^{*, C}\left(c_{i}\right)$, another solver's performance is the following random variable: $\widetilde{v}^{*, C} \equiv v^{*, C}(\widetilde{c})=$ $v^{*, P}(\widetilde{a})=\widetilde{v}^{*, P}$. Then, under $r(e)=\theta e$, solver $i^{\prime}$ s problem (3) in a cost-based project becomes

$$
\max _{v_{i}} \sum_{j=1}^{n} A_{j} \frac{(n-1) !}{(j-1) !(n-j) !} P\left(v_{i}>\widetilde{v}^{*, C}\right)^{n-j} P\left(v_{i} \leq \widetilde{v}^{*, C}\right)^{j-1}-c_{i} \frac{v_{i}}{\theta}-k
$$




$$
=\max _{v_{i}} \sum_{j=1}^{n} A_{j} \frac{(n-1) !}{(j-1) !(n-j) !} P\left(v_{i}>\widetilde{v}^{*, P}\right)^{n-j} P\left(v_{i} \leq \widetilde{v}^{*, P}\right)^{j-1}-c \frac{v_{i}}{a_{i} \theta}-k,
$$

where the equality follows because $\widetilde{v}^{*, C}=\widetilde{v}^{*, P}$ and $c_{i}=\eta^{-1}\left(a_{i}\right)=c /\left(\theta a_{i}\right)$. Thus, under $r(e)=\theta e$, the solver's problem (EC.2) in a cost-based project is equivalent to the solver's problem (EC.3) in a productivity-based project. As a result, given that all other solvers have performance $v^{*, P}\left(a_{i}\right)=$ $v^{*, C}\left(c_{i}\right)$, by using the mapping $\eta$, we obtain the same best response for solver $i$ under cost-based and productivity-based projects. This shows that the equilibrium performance under cost-based and productivity-based projects satisfies $v^{*, P}\left(a_{i}\right)=v^{*, P}\left(\eta\left(c_{i}\right)\right)=v^{*, C}\left(c_{i}\right)=v^{*, C}\left(c /\left(\theta a_{i}\right)\right)$. Finally, using $\widetilde{a}=\eta(\widetilde{c})=c /(\theta \widetilde{c})$, we obtain

$$
H\left(a_{i}\right)=P\left(\widetilde{a} \leq a_{i}\right)=P\left(c /(\theta \widetilde{c}) \leq a_{i}\right)=P\left(c /\left(\theta a_{i}\right) \leq \widetilde{c}\right)=1-G\left(c /\left(\theta a_{i}\right)\right)
$$

(b) In an expertise-based project, all solvers except solver $i$ have performance based on $v^{*, E}\left(\beta_{i}\right)$. We will construct a bijective mapping $\omega: R_{+} \rightarrow R_{+}$from a solver's expertise $\beta_{i}$ to a productivity $a_{i}$ (i.e., $\left.\omega\left(\beta_{i}\right)=a_{i}\right)$ such that given that all other solvers have performance $v^{*, P}\left(a_{i}\right)=v^{*, E}\left(\beta_{i}\right)$, solver $i$ will have the same best-response performance. Define solver $i$ 's productivity as $a_{i}=$ $\omega\left(\beta_{i}\right)=\exp \left(\beta_{i} / \theta\right)$. Given $v^{*, P}\left(a_{i}\right)=v^{*, E}\left(\beta_{i}\right)$, another solver's performance is the following random variable: $\widetilde{v}^{*, E} \equiv v^{*, E}(\widetilde{\beta})=v^{*, P}(\widetilde{a})=\widetilde{v}^{*, P}$. Then, under $r(e)=\theta \log e$, solver $i^{\prime}$ s problem (3) in an expertise-based project becomes

$$
\begin{aligned}
& \max _{v_{i}} \sum_{j=1}^{n} A_{j} \frac{(n-1) !}{(j-1) !(n-j) !} P\left(v_{i}>\widetilde{v}^{*, E}\right)^{n-j} P\left(v_{i} \leq \widetilde{v}^{*, E}\right)^{j-1}-c \exp \left(\left(v_{i}-\beta_{i}\right) / \theta\right)-k \\
= & \max _{v_{i}} \sum_{j=1}^{n} A_{j} \frac{(n-1) !}{(j-1) !(n-j) !} P\left(v_{i}>\widetilde{v}^{*, P}\right)^{n-j} P\left(v_{i} \leq \widetilde{v}^{*, P}\right)^{j-1}-c \frac{\exp \left(v_{i} / \theta\right)}{a_{i}}-k,
\end{aligned}
$$

where the equality follows because $\widetilde{v}^{*, E}=\widetilde{v}^{*, P}$ and $\beta_{i}=\omega^{-1}\left(a_{i}\right)=\theta \log \left(a_{i}\right)$. Thus, under $r(e)=$ $\theta \log e$, the solver's problem (EC.4) in an expertise-based project is equivalent to the solver's problem (EC.5) in a productivity-based project. As a result, given that all other solvers have performance based on $v^{*, P}\left(a_{i}\right)=v^{*, E}\left(\beta_{i}\right)$, by using the the mapping $\omega$, we obtain the same best-response for solver $i$ under an expertise-based project as that under a productivity-based project. This shows that the equilibrium performance under expertise-based and productivity-based projects 
satisfies $v^{*, P}\left(a_{i}\right)=v^{*, P}\left(\omega\left(\beta_{i}\right)\right)=v^{*, E}\left(\beta_{i}\right)=v^{*, E}\left(\theta \log \left(a_{i}\right)\right)$. Finally, using the mapping $\widetilde{a}=\omega(\widetilde{\beta})=$ $\exp (\widetilde{\beta} / \theta)$, we obtain

$$
H\left(a_{i}\right)=P\left(\widetilde{a} \leq a_{i}\right)=P\left(\exp (\widetilde{\beta} / \theta) \leq a_{i}\right)=P\left(\widetilde{\beta} \leq \theta \log \left(a_{i}\right)\right)=F\left(\theta \log \left(a_{i}\right)\right)
$$

